INPLASY PROTOCOL

To cite: Cui et al. Effectiveness of Acupotomy Lysis for Infrapatellar Fat Pad to Treat Infrapatellar Fat Pad Injury: A Meta-analysis. Inplasy protocol 2020120085. doi:

10.37766/inplasy2020.12.0085

Received: 16 December 2020

Published: 16 December 2020

Corresponding author:

Cui Shaoyang

416846554@qq.com

Author Affiliation:

Shenzhen Hospital of Guangzhou University of

Chinese Medicine

Support: Sanming Project of Medicine.

Review Stage at time of this submission: Preliminary searches.

Conflicts of interest: None.

\section{Effectiveness of Acupotomy Lysis for Infrapatellar Fat Pad to Treat Infrapatellar Fat Pad Injury: A Meta-analysis}

Cui, SY1; Fang, ZJ², Lin, R3; Xu, MZ4; Luo, J5.

Review question / Objective: Infrapatellar fat pad injury is difficult to heal, the course of disease is longer (usually more than one year) can appear the knee peripheral muscle group atrophy to varying degrees, at the same time may appear the knee joint force line change, causes the ankle joint, the hip joint, even the spine deviation, causes the whole body a series of problems, may seriously affect the patient's work and the quality of life. Many knee joint lesions, such as degenerative osteoarthritis, can show the clinical manifestations of infrapatellar fat pad injury. According to the latest domestic and foreign studies, infrapatellar fat pad inflammation is an important pathological basis of knee osteoarthritis is an early change of knee degenerative disease, its incidence accounts for more than $80 \%$ of knee disease. Acupotomy is a special acupuncture method developed in recent decades. It can prolong and amplify the effect of acupuncture to some extent by loosening local soft tissue. A lot of clinical studies in recent years have found that the treatment of infrapatellar fat pad injury with acupotomy has better curative effect, less times of treatment and small side effects than other methods, but the sample size is small, and there is no convincing evidence. Therefore, this paper uses the method of systematic evaluation to evaluate the clinical trial of acupotomy loosening infrapatellar fat pad in the treatment of infrapatellar fat pad injury, in order to provide more evidence-based evidence for the application of this method in the treatment of knee joint diseases.

INPLASY registration number: This protocol was registered with the International Platform of Registered Systematic Review and Meta-Analysis Protocols (INPLASY) on 16 December 2020 and was last updated on 16 December 2020 (registration number INPLASY2020120085).

\section{INTRODUCTION}

Review question / Objective: Infrapatellar fat pad injury is difficult to heal, the course of disease is longer (usually more than one year) can appear the knee peripheral muscle group atrophy to varying degrees, 
at the same time may appear the knee joint force line change, causes the ankle joint, the hip joint, even the spine deviation, causes the whole body a series of problems, may seriously affect the patient's work and the quality of life. Many knee joint lesions, such as degenerative osteoarthritis, can show the clinical manifestations of infrapatellar fat pad injury. According to the latest domestic and foreign studies, infrapatellar fat pad inflammation is an important pathological basis of knee osteoarthritis is an early change of knee degenerative disease, its incidence accounts for more than $80 \%$ of knee disease. Acupotomy is a special acupuncture method developed in recent decades. It can prolong and amplify the effect of acupuncture to some extent by loosening local soft tissue. A lot of clinical studies in recent years have found that the treatment of infrapatellar fat pad injury with acupotomy has better curative effect, less times of treatment and small side effects than other methods, but the sample size is small, and there is no convincing evidence. Therefore, this paper uses the method of systematic evaluation to evaluate the clinical trial of acupotomy loosening infrapatellar fat pad in the treatment of infrapatellar fat pad injury, in order to provide more evidence-based evidence for the application of this method in the treatment of knee joint diseases.

Condition being studied: Infrapatellar fat pad injury is difficult to heal, the course of disease is longer (usually more than one year) can appear the knee peripheral muscle group atrophy to varying degrees, at the same time may appear the knee joint force line change, causes the ankle joint, the hip joint, even the spine deviation, causes the whole body a series of problems, may seriously affect the patient's work and the quality of life. Many knee joint lesions, such as degenerative osteoarthritis, can show the clinical manifestations of infrapatellar fat pad injury. Acupotomy is a special acupuncture method developed in recent decades. It can prolong and amplify the effect of acupuncture to some extent by loosening local soft tissue. A lot of clinical studies in recent years have found that the treatment of infrapatellar fat pad injury with acupotomy has better curative effect, less times of treatment and small side effects than other methods, but the sample size is small, and there is no convincing evidence.

\section{METHODS}

Search strategy: "Needle knife "[ title: intelligence] AND(" subpatellar fat pad " or "hoffa"[ title: intelligence]).

Participant or population: Patients with subpatellar fat pad.

Intervention: Use needle knife to release the treatment of subpatellar fat pad.

Comparator: use non-needle knife treatment.

Study designs to be included: randomized controlled trials (RCTs) on the acupotome to loosen the infrapatellar fat pad for the treatment of infrapatellar fat pad injury.

Eligibility criteria: Conforms to the Diagnostic Criteria of Subpatellar Fat Pad Injury [5] Guidelines for Clinical Diagnosis and Treatment, with unlimited sex, age and source of cases.

Information sources: We searched databases including CBM, CNKI, VIP, WangFang Data, Pubmed, Embase, The Cochrane Library and MEDLINE.

Main outcome(s): 1 - treatment efficiency. 2 - VAS score.

Quality assessment / Risk of bias analysis: The quality of RCTs were evaluated by Cochrane Risk of Bias Asssment Tool and the RevMan5.4.1 software was used for meta-analyses.

Subgroup analysis: None.

Sensibility analysis: None.

Country(ies) involved: China. 
Keywords: infrapatellar fat pad injury; Acupotomy; Meta-analysis; Systematic review; Randomized controlled trial.

Contributions of each author:

Author 1 - Cui Shaoyang.

Author 2 - Fang Zijie.

Author 3 - Lin Run.

Author 4 - Xu Mingzhu.

Author 5 - Luo Jing. 